\title{
Shoulder pain due to cervical radiculopathy: an underestimated long-term complication of herpes zoster virus reactivation?
}

\author{
Stefano Gumina ${ }^{1} \cdot$ Vittorio Candela $^{1}$ (D) Daniele Passaretti $^{1} \cdot$ Ciro Villani $^{1}$
}

Received: 8 April 2017 / Accepted: 24 July 2017

(C) SICOT aisbl 2017

\begin{abstract}
Purpose To evaluate if herpes zoster virus (HZV) reactivation may be considered in the aetiology of cervical radiculopathy.

Methods The study group was composed of 110 patients (52 M-58F; mean age \pm SD:46.5 \pm 6.12 ; range:40-73) with a clinical diagnosis of cervical radiculopathy. Patients with signs of chronic damage on neurophysiological studies were submitted to an X-ray and to an MRI of the cervical spine in order to clarify the cause of the cervical radiculopathy and were investigated for a possible reactivation of HZV; HZV reactivation was considered as "recent" or "antique" if it occurs within or after 24 months from the onset of symptoms, respectively. Data were submitted to statistics.

Results Thirty-eight patients (34,5\%,16 M-22F) had a history of HZV reactivation: four ( $2 \mathrm{M}-2 \mathrm{~F})$ were "recent" and 34 (14 M-20F) were "antique". In 68 of 110 participants $(61,8 \%, 30 \mathrm{M}-38 \mathrm{~F})$, pathological signs on X-ray and/or MRI of the cervical spine appeared; in the remaining 42 $(38,2 \%, 22 \mathrm{M}-20 \mathrm{~F}) \mathrm{X}$-ray and MRI resulted as negative. Among patients with HZV reactivation, seven $(18,4 \%)$ had a "positive" X-ray-MRI while in $31(81,6 \%)$ the instrumental exams were considered as negative. The prevalence of "antique" HZV reactivations was statistically greater in the group of patients with no pathological signs on X-ray/MRI of the cervical spine with respect to the group with a pathological instrumental exam $(p<0.01)$.
\end{abstract}

Vittorio Candela

Vittorio.candela@yahoo.it

1 Department of Orthopaedics and Traumatology, Shoulder and Elbow Unit, Sapienza, University of Rome, Rome, Italy
Conclusions It may be useful to investigate the presence of a positive history of HZV reactivation and to consider it as a long-term complication of a cervical root inflammation especially in patients in which X-ray and MRI of the cervical spine did not show pathological findings.

Keywords Shoulder pain $\cdot$ Shoulder pathology $\cdot$ Herpes zoster virus $\cdot$ Cervical radiculopathy $\cdot$ Neck pain $\cdot$ Zoster reactivation $\cdot$ Cervical spine disease

\section{Introduction}

Herpes zoster is a viral disease characterized by a painful skin rash composed of vescicles located in a restricted area on one side of the body (left or right), often in a stripe. The initial infection with herpes zoster virus (HZV) causes the acute, short-lived illness, known as chickenpox, which normally occurs in children and young adults. After the initial episode, HZV remains latent in the nerve cell bodies and, less frequently, in the non-neuronal satellite cells of the dorsal root, cranial nerve or autonomic ganglia, without causing any symptoms $[1,2]$. Years after the initial infection, the virus may spread from one or more ganglia along nerves of an affected segment and infect the corresponding dermatome (an area of skin supplied by one spinal nerve) causing a painful skin rash [3, 4].

Although herpes zoster reactivation typically resolves within three to five weeks, some complications may appear: secondary bacterial infection; motor involvement, including weakness especially in "motor herpes zoster"; eye involvement (trigeminal nerve involvement); and especially postherpetic neuralgia. The latter is a nerve pain due to damage caused by the virus responsible for sending abnormal electrical signals to the brain; these signals may convey excruciating pain, and may persist or recur for months, years, or 
for life. Typically, the neuralgia is confined to a dermatomic area of the skin and follows an outbreak of herpes zoster in that same dermatomic area; it begins when the herpes zoster blisters have crusted over and begun to heal, but it can also begin after some months from the disappearance of the lesions or in the absence of herpes zoster.

We noted a history of a reactivation of herpes zoster in many patients with a clinical diagnosis of cervical radiculopathy. On the basis of these observations, the aims of our study were: a) to assess the prevalence of a positive history of HZV reactivation in patients with a diagnosis of cervical radiculopathy; b) to evaluate if HZV may be considered as an aetiological factor of cervical radiculopathy especially in patients with no pathological signs on X-ray and MRI of the neck.

\section{Materials and methods}

An observational design study was used. The study group was composed initially of 185 consecutive patients with a clinical diagnosis of cervical radiculopathy enrolled in the outpatient clinic of our hospital between January 2014 and October 2015.

The diagnosis of cervico-brachialgia was made on the basis of an accurate physical examination, performed by the most expert of the Authors (SG), including: evaluation of range of motion (ROM); four clinical tests with an high accuracy for cervical radiculopathy: Spurling test, shoulder abduction test, Valsalva manueuver, traction/neck distraction test [5-10], Arm Squeeze test [11] and motor, sensory and reflex examination. Inclusion criteria for all participants were: clinical diagnosis of cervical radiculopathy; onset of disturbs from less than six months; age $\geq 40$ years; signs of chronic damage on needle electromyography (EMG) and nerve conduction studies (NCS). Patients with the following conditions were excluded from the study design: systemic disease known to be responsible for a generalized peripheral neuropathy; history of surgical procedure for pathologies giving rise to neck pain; disease that can lead to a pathological narrowing of the vertebral canal.

After physical examination, patients were all submitted to an X-ray (antero-posterior, lateral and oblique); to an MRI (Siemens Magnetom Avanto Medical $76 \times 32,3 \mathrm{~T}$; repetition time, 3200 milliseconds; echo time, 85 milliseconds) of the cervical spine in the Radiology Centre of our University in order to clarify the cause of the cervical radiculopathy. Before the instrumental examination, all participants were investigated about a possible reactivation of HZV infection: they were asked if they suffered from a first infection in the past (when they were younger) and if there was a reactivation of the virus. Arbitrarily we considered a HZV reactivation as "recent" and "antique" if it occurs within or after 24 months from the onset of symptoms/pain, respectively.

Regarding the evaluation of the MRI, the imaging protocol consisted of sagittal fast spin echo T1 and T2 weighted images. Section thickness was $3 \mathrm{~mm}$.

Following the pattern of Modic et al. [12, 13] and Frobin et al. [14], the most expert of the Authors (SG) assessed three items:

A) Nucleus signal classified (T2-weighted image) as: (1) no signal loss, (2) moderate signal loss and (3) total loss;

B) Prolapse classified (T1-weighted image) as: (1) no prolaps, (2) prolaps;

C) Bone marrow signal of adjacent vertebral body (T1-and T2-weigheted image) classified as: (1) no intensity change and (2) intensity change.

This classification results in 12 degenerative categories, from A to L (Table 1).

A lateral radiographic view of the cervical spine was obtained from each patient. Normal disc height has been compiled by applying the distortion compensated Roentgen analysis (DCRA) protocol on lateral radiographs taken in extension in a normal cohort $[15,16]$. The angle corrected disc height in the participant case was compared to the appropriate segmental level, age and gender. Physiological disc height is on the order of 0.35 , i.e. disc height amounts to $1 / 3$ of the vertebral height in segments $\mathrm{C} 2 / \mathrm{C} 3$ to $\mathrm{C} 6 / \mathrm{C} 7[15,16]$. Arbitrarily, we considered as "negative" the MRIs that fell into class A and the X-rays in which there was no narrowing of the intervertebral disc height.

All participants signed an informed consent form in accordance with the Declaration of Helsinki. According to the law of our country, this study does not need any ethical committee approval.

Table 1 Classification of cervical spine degeneration on MRI

\begin{tabular}{clll}
\hline Categories & Nucleus signal & Bone marrow signal & Prolapse \\
\hline A & No signal loss & No intensity change & No prolapse \\
B & No signal loss & No intensity change & Prolapse \\
C & No signal loss & Intensity change & No prolapse \\
D & No signal loss & Intensity change & Prolapse \\
E & Moderate signal loss & No intensity change & No prolapse \\
F & Moderate signal loss & No intensity change & Prolapse \\
G & Moderate signal loss & Intensity change & No prolapse \\
H & Moderate signal loss & Intensity change & Prolapse \\
I & Total signal loss & No intensity change & No prolapse \\
J & Total signal loss & No intensity change & Prolapse \\
K & Total signal loss & Intensity change & No prolapse \\
L & Total signal loss & Intensity change & Prolapse \\
\hline
\end{tabular}




\section{Statistical analysis}

Statistical Package for Social Sciences (SPSS) 116 version 18 was used for calculations. All the data were analysed by a single blinded researcher. Computed $P$ values were 2 -sided, and a $p<0.05$ was used to determine statistical significance.

The Chi-squared test was used to evaluate the differences in prevalence of HZV reactivations between patients with "positive" and "negative" instrumental examinations.

\section{Results}

The study group was finally composed of 110 patients (52 M58F; mean age \pm SD: $46.5 \pm 6.12$; range 40-73).

Thirty-eight patients $(34.5 \%, 16 \mathrm{M}-22 \mathrm{~F})$ had a positive history of HZV reactivation; among them, four reactivations (2 M-2F) were considered as "recent" since they occurred less than 24 months before the check; 34 (14 M-20F) were antique (> 24 months).

In 68 of 110 participants $(61.8 \%, 30 \mathrm{M}-38 \mathrm{~F})$, we found pathological signs on X-ray and/or MRI of the cervical spine; degeneration categories on MRI, according to the classification we used, and the presence of narrowing of the intervertebral disc height on X-ray are reported in Table 2. In the remaining $42(38.2 \%, 22 \mathrm{M}-20 \mathrm{~F})$ both X-ray and MRI did not justify the clinical findings.

No significant difference was found between prevalence of "positive" and "negative" results of MRI/X-ray $(p>0.05)$.

Among the group of patients with HZV reactivation $(n=38)$, seven (18.4\%; two "recent", five "antiques") had a "positive" X-ray-MRI while in 31 (81.6\%; two "recent", 29 "antiques") the instrumental exams were considered as negative.

We found that the prevalence of "antique" HZV reactivations was statistically greater in the group of patients with no pathological signs on X-ray/MRI of the cervical spine with respect to the group with a pathological instrumental exam $(p<0.01)$.

\section{Discussion}

Cervical radiculopathy is a disorder of the cervical spine nerve root [17]; its yearly incidence is about $0.1 \%$ [18] and neck

Table 2 Results of the instrumental exams in the study group

\begin{tabular}{lll}
\hline$N^{\circ}$ & MRI categories & $\begin{array}{l}\text { Alterations on } \\
\text { X-ray }\end{array}$ \\
\hline 42 & A & No \\
12 & E & Yes \\
39 & F & Yes \\
10 & G & Yes \\
7 & H & Yes \\
\hline
\end{tabular}

pain radiating to the arm and fingers corresponding to the dermatomes involved, paraesthesias in arm and hand connected to diminished muscle tendon reflex, sensory disturbances and/or motor weakness with dermatomal/myotomal distribution represents the classic clinical picture [19]. Two studies on 100 and 561 patients $[18,20]$, respectively, reported that the classic figure is not always present: paraesthesias were detected in $91 \%$ and $89.7 \%$, respectively, sensory loss in $24 \%$ and $33 \%$, symptoms of weakness in $34 \%$ and $15.3 \%$, signs of weakness in $70 \%$ and $64.2 \%$, and hypoactive tendon reflex in $72 \%$ and $84.1 \%$, respectively. C7 is the most affected cervical spinal root, followed by C6, C5, C4 and C8 [20].

In most cases, it is caused by a cervical disc herniation or other space-occupying lesion, resulting in nerve root inflammation, impingement or both [17, 21]. Other less common causes (Sarcoidosis [22], vascular decompression of a vertebral artery loop [23], peripheral neuropathy [24], athetoid cerebral palsy [25], previous anterior cerebral arthrodesis [26], OJ syndrome [27], trauma [28], Non-Hodgkin's lymphoma [29], giant cell arteritis [30], cysts [31] Pancoast tumor [32] have also been reported. To our knowledge, little information is available regarding the reactivation of HZV as a possible cause of cervical root inflammation; on the other hand, many studies [1-4] have considered HZV as an etiological factor responsible for neuropathic pain. The physiopathological mechanism is not completely clear even if it is known that a key factor in the neural plasticity underlying neuropathic pain alters gene expression in sensory root ganglia neurons. Injury to sensory nerves induces neurochemical, physiological and anatomical modifications to afferent and central neurons, such as afferent terminal sprouting and inhibitory interneuron loss. Following nerve damage, $\mathrm{NaCl}$ channels accumulation cause hyperexcitability, and downregulation of the TTX-resistant Nav1.8 (sensory neuron specific, SNS1) channel and upregulation of TTX-sensitive Nav1.3 (brain type III) and TRPV1 channels. These changes are responsible for the increasing of NMDA glutamate receptor-dependent excitability of spinal horn neurons and are restricted to the ipsilateral (injured) side [33].

This is the first study performed with the aim to evaluate the real prevalence of a HZV reactivation in patients with cervical radiculopathy. In our series, composed of 110 patients, we found that the prevalence of an antique HZV reactivation was statistically higher in patients with no pathological signs on MRI/X-ray of the neck with respect to those with abnormalities on instrumental exams.

We decided to divide antique and recent $\mathrm{HZV}$ reactivations and to consider only antique infections in the statistical analysis because the clinical figure of a recent virus reactivation (dermatological and/or neurological) could include cervical root inflammation as a short-term complication; patients with reactivation occurred more than 24 months ago have been considered because, in this case, we are sure they presented 
a free interval from the disease and so it may be plausible to consider cervical root inflammation as a long term complication of $\mathrm{HZV}$.

These findings assume an important diagnostic and therapeutic value; in fact, it may be useful to investigate the presence of a positive history of $\mathrm{HZV}$ reactivation and to consider it as a longterm complication of a cervical root inflammation especially in patients in which X-ray and MRI of the cervical spine did not show pathological findings that would justify the clinical figure.

Moreover, these results could change the therapeutic approach to this disease, with the real possibility of adding an antiretroviral treatment to the common conservative treatment consisting of physiotherapy and/or drugs for pain and inflammation.

It would be useful to perform prospective randomized study with the aim to evaluate the effectiveness of antiretroviral treatment in patients with a clinical diagnosis of cervical root inflammation and a positive history of HZV reactivation.

\section{Compliance with ethical standards}

Conflict of interest On behalf of all authors, the corresponding author states that there is no conflict of interest.

Funding No funding has been received.

\section{References}

1. Johnson RW, Dworkin RH (2003) Clinical review: treatment of herpes zoster and postherpetic neuralgia. BMJ 326(7392):748750. doi:10.1136/bmj.326.7392.748 PMC 1125653

2. Kennedy PG (2002) Varicella-zoster virus latency in human ganglia. Rev Med Virol 12(5):327-334. doi:10.1002/rmv.362

3. Peterslund NA (1991) Herpesvirus infection: an overview of the clinical manifestations. Scand J Infect Dis Suppl 80:15-20

4. Gilden DH, Cohrs RJ, Mahalingam R (2003) Clinical and molecular pathogenesis of varicella virus infection. Viral Immunol 16(3): 243-258. doi:10.1089/088282403322396073

5. Shah KC, Rajshekhar V (2004) Reliability of diagnosis of soft cervical disc prolapse using Spurling's test. Br J Neurosurg 18:480-483

6. Wainner RS, Fritz JM, Irrgang JJ, Boninger ML, Delitto A, Allison S (2003) Reliability and diagnostic accuracy of the clinical examination and patient self-report measures for cervical radiculopathy. Spine 28:52-62

7. Viikari-Juntura E, Porras M, Laasonen EM (1989) Validity of clinical tests in the diagnosis of root compression in cervical disc disease. Spine 14:235-257

8. Tong HC, Haig AJ, Yamakawa K (2002) The Spurling test and cervical radiculopathy. Spine 27:156-159

9. Sandmark H, Nisell R (1995) Validity of five common manual neck pain provoking tests. Scand J Rehabil Med 27:131-136

10. Shabat S, Leitner Y, David R, Folman Y (2012) The correlation between Spurling test and imaging studies in detecting cervical radiculopathy. J Neuroimaging 22(4):375-378. doi:10.1111/j. 1552-6569.2011.00644

11. Gumina S, Carbone S, Albino P, Gurzi M, Postacchini F (2013) Arm squeeze test: a new clinical test to distinguish neck from shoulder pain. Eur Spine J 22(7):1558-1563. doi:10.1007/s00586-013-2788-3
12. Modic MT, Masaryk TJ, Ross JS, Carter JR (1998) Imaging of the degenerative disc disease. Radiology 168:177-186

13. Modic MT (1989) Degenerative disorders of the spine. In: Masaryk TJ, Ross JS (eds) Magnetic resonance imaging of the spine, 2nd edn. Mosby, St. Louis, pp 80-150

14. Frobin W, Brinckmann P, Kramer M, Hartwig E (2001) Height of lumbar discs measured from radiographs compared with degeneration and height classified from MR images. Eur Radiol 11:263-269

15. Frobin W, Leivseth G, Biggemann M, Brinckmann P (2002) Sagittal plane motion of the cervical spine a new precision measurement protocol and normal motion data of healthy adults. Clin Biomech 17:21-31

16. Frobin W, Leivseth G, Biggemann M, Brinckmann P (2002) Vertebral height, disc height, posterior displacement and densatlas gap in the cervical spine: precision measurement protocol and normal data. Clin Biomech 17:423-431

17. Radhakrishnan K, Litchy WJ, O'Fallon WM, Kurland LT (1994) Epidemiology of cervical radiculopathy. A population-based study of Rochester, Minnesota, 1976 through 1990. Brain 117(Pt 2):325-335

18. Goodman CC, Snyder TE (1995) Differential diagnosis in physical therapy. Saunders, Philadelphia

19. Yoss RE, Corbin KB, Maccarty CS, Love JG (1957) Significance of symptoms and signs in localization of involved root in cervical disk protrusion. Neurology 7:673-683

20. Abbed KM, Coumans JV (2007) Cervical radiculopathy: pathophysiology, presentation, and clinical evaluation. Neurosurgery 60(1 supp1 1):28-34

21. Kuijper B, Tans JT, Schimsheimer RJ, van der Kallen BF, Beelen A, Nollet F, de Visser M (2009) Degenerative cervical radiculopathy: diagnosis and conservative treatment. A review Eur J Neurol 16(1): 15-20. doi:10.1111/j.1468-1331.2008.02365

22. Atkinson R, Ghelman B, Tsairis P, Warren RF, Jacobs B, Lavyne M (1982) Sarcoidosis presenting as cervical radiculopathy: a case report and literature review. Spine 7:412-416

23. Detwiler PW, Porter RW, Harrington TR, Sonntag VK, Spetzler RF (1998) Vascular decompression of a vertebral artery loop producing cervical radiculopathy: case report. J Neurosurg 89:485-488

24. Dyck P, Thomas P (1984) Peripheral neuropathy, vol 1 and 2. Saunders, Philadelphia

25. Fujy T, Yonenobu K, Fujiwara K (1987) Cervical radiculopathy or myelopathy secondary to athetoid cerebral palsy. J Bone Joint Surg (Am) 69:815-821

26. Hilibrand AS, Carlson GD, Palumbo MA, Jones PK, Bohlman HH (1999) Radiculopathy and mielopathy at segment adjacent to the site of a previous anterior cervical arthrodesis. J Bone Joint Surg (Am) 81:519-528

27. Le Ban MM, Braker AM, Meerschaert JR (1989) Airport-induced cervical traction: Radiculopahty: the OJ syndrome. Arch Phys Med Rehabil 70:845-847

28. Makela JP, Hietaniemi K (1997) Neck injury after repeated flexions due to parachuting. Aviat Space Environ Med 68:228-229

29. Perez-Martienz DA, Saiz-Diaz RA, de Toledo M, Berbel A, Martínez-Salio A, Prieto S (1998) Cervical radiculopathy as a form of presentation of non-Hodgkin's lymphoma. Rev Neurol 27:91-93

30. Sanchez MC, Arenillas JIC, Gutierrez DA, Gutierrez Alonso JL, de Portugal AJ (1983) Cervical radiculopathy: a rare symptom of giant cell arteritis. Arthritis Rheumatol 26:207-209

31. Uematsu Y, Tokuhashi Y, Matsuzaki H (1998) Radiculopathy after laminoplasty of the cervical spine. Spine 23:2057-2062

32. Vargo MM, Flood KM (1990) Pancoast tumor presenting as cervical radiculopathy. Arch Phys Med Rehabil 71:606-609

33. Steiner I, Kennedy PG, Pachner AR (2007) The neurotropic herpes viruses: herpes simplex and varicella-zoster. Lancet Neurol 6(11): 1015-1028. doi:10.1016/S1474-4422(07)70267-3 